\title{
Correspondence: SEMA4A variation and risk of colorectal cancer
}

Ben Kinnersley ${ }^{1}$ Daniel Chubb¹, Sara E. Dobbins ${ }^{1}$, Matthew Frampton ${ }^{1}$, Stephan Buch ${ }^{2}$, Maria N. Timofeeva ${ }^{3}$, Sergi Castellví-Bel ${ }^{4}$, Susan M. Farrington ${ }^{3}$, Asta Forsti ${ }^{5}$, Jochen Hampe ${ }^{6}$, Kari Hemminki ${ }^{5}$, Robert M.W. Hofstra 7,8 , Emma Northwood ${ }^{9}$, Claire Palles ${ }^{10}$, Manuela Pinheiro ${ }^{11}$, Clara Ruiz-Ponte ${ }^{12}$, Clemens Schafmayer ${ }^{13}$, Manuel R. Teixeira ${ }^{11}$, Helga Westers ${ }^{8}$, Tom van Wezel ${ }^{14}$, D. Timothy Bishop ${ }^{9}$, Ian Tomlinson ${ }^{10}$, Malcolm G. Dunlop ${ }^{3} \&$ Richard S. Houlston ${ }^{1}$

Nature Communications 7:10611 doi: 10.1038/ncomms10611 (2016); Published 10 Mar 2016

Sill and co-workers ${ }^{1}$ report that germline variation in semaphorin 4A (SEMA4A) influences colorectal cancer (CRC) risk. This stems from identifying the SEMA4A p.Val78Met variant in one kindred with familial colorectal cancer type $\mathrm{X}$ (FCCTX) and subsequently p.Gly484Ala (c.1451G $>$ C, rs148744804) and p.Ser326Phe (c.977C > T) mutations along with the single nucleotide polymorphism (SNP) p.Pro682Ser (c.2044C > T, rs76381440) among an additional 53 FCCTX cases. In comparing the frequency of rs76381440 genotype in 47 FCCTX cases and 1,138 controls, c.2044T carrier status was reported to be associated with 6.79 -fold increased CRC risk ${ }^{1}$.

Here, we report a well-powered study that casts doubt on SEMA4A as a CRC predisposition gene. This has important implications for clinical genetics because inappropriate screening or intervention might be recommended to carriers.

First, we studied the contribution of the recurrent variants, rs148744804 and rs76381440, to CRC analysing 6,856 CRC cases and 10,090 controls from six European populations as previously described $^{2,3}$. These comprised (1) 3,666 English case patients ( $n=250$ from the CORGI study; $n=957$ from the QUASAR study; $n=1,168$ from NSCCG; $n=1,291$ from a Leeds casecontrol series) and 6,140 control patients ( $n=5,694$ from the 1958 Birth Cohort; $n=446$ from the Leeds series); (2) 2,052 Scottish CRC cases and 2,004 Scottish controls $(n=1,452$ from the 1935 and 1928 Lothian birth cohorts; $n=552$ from generation Scotland); (3) 276 Spanish cases and 284 controls; (4) 800 Dutch samples $(n=337$ Leiden cases and $n=337$ controls; $n=74$ Groningen cases and $n=52$ controls); (5) 199 Portuguese cases and 186 controls and (6) 1,339 German samples ( $n=77$ Heidelberg cases and $n=88$ controls; $n=175$ Kiel cases and $n=999$ controls). Collectively these samples provide $>99 \%$ power $(\alpha=0.05)$ to detect the lower limit of the point estimate reported by Sill and co-workers for the association between p.Pro682Ser and $\mathrm{CRC}^{1}$ ) (odds ratio $[\mathrm{OR}]=2.6)$.

We used Infinium HumanExome BeadChips (Illumina San Diego, CA) to genotype our samples as previously described ${ }^{2,3}$ and extracted the genotypes for rs148744804 and rs76381440. We validated genotyping by sequencing 541 random samples, providing very strong concordance $\left(r^{2}=1.0\right.$ and 0.99 for rs148744804 and rs76381440, respectively; Supplementary Table 1). We used principal component analysis to confirm ancestral comparability of cases and controls (Supplementary Figure 1).

None of the six series showed a statistically significant difference in frequency of rs148744804 or rs76381440 genotype between cases and controls (Table 1). In a meta-analysis of data from all studies, we found no association between c.1451C or c.2044T carrier status and CRC $(\mathrm{OR}=1.14,95 \%$ confidence

\footnotetext{
${ }^{1}$ Division of Genetics and Epidemiology, The Institute of Cancer Research, Surrey, Sutton SM2 5NG, UK. ${ }^{2}$ Department of Medicine I, University Hospital Dresden, Dresden 23538, Germany. ${ }^{3}$ Colon Cancer Genetics Group, Institute of Genetics and Molecular Medicine, University of Edinburgh and Medical Research Council (MRC) Human Genetics Unit, Edinburgh EH4 2XU, UK. ${ }^{4}$ Department of Gastroenterology, Hospital Clínic, Centro de Investigación Biomédica en Red de Enfermedades Hepáticas y Digestivas, Institut d'Investigacions Biomèdiques August Pi i Sunyer, University of Barcelona, Catalonia, Barcelona 8036, Spain. ${ }^{5}$ German Cancer Research Center, Heidelberg 69120, Germany. ${ }^{6}$ Department of Internal Medicine I, Hospital Schleswig-Holstein, Kiel 24105, Germany. ${ }^{7}$ Department of Clinical Genetics, Erasmus Medical Center, Rotterdam 3000 CA, The Netherlands. ${ }^{8}$ University of Gronigen, University Medical Centre Gronigen, Department of Genetics, Gronigen 9700 RB, The Netherlands. ${ }^{9}$ Section of Epidemiology and Biostatistics, Leeds Institute of Cancer and Pathology, University of Leeds, Leeds LS9 7TF, UK. ${ }^{10}$ Molecular and Population Genetics Laboratory, Wellcome Trust Centre for Human Genetics, University of Oxford, Oxford OX3 7BN, UK. ${ }^{11}$ Department of Genetics, Portugese Oncology Institute, Porto 4200-072, Portugal. ${ }^{12}$ Galician Public Foundation of Genomic Medicine, Centro de Investigación Biomédica en Red de Enfermedades Rares, Genomics Medicine Group, Hospital Clínico, Santiago de Compostela, University of Santiago de Compostela, Galicia 15782, Spain. ${ }^{13}$ Department of General and Thoracic Surgery, University Hospital SchleswigHolstein, Kiel 24105, Germany. ${ }^{14}$ Leiden Department of Pathology, Leiden University Medical Center, Leiden, 2333 ZA, The Netherlands. Correspondence and requests for materials should be addressed to R.S.H. (email: richard.houlston@icr.ac.uk).
} 


\begin{tabular}{|c|c|c|c|c|c|c|c|c|c|c|c|c|}
\hline Study & \multicolumn{5}{|c|}{ Case } & \multicolumn{5}{|c|}{ Control } & OR $(95 \% \mathrm{Cl})$ & $\boldsymbol{P}$ \\
\hline \multicolumn{13}{|l|}{ (A) rs76381440 } \\
\hline England and Wales & 0 & 152 & 3,514 & 3,666 & 0.021 & 2 & 259 & 5,879 & 6,140 & 0.021 & $0.97(0.79-1.20)$ & 0.80 \\
\hline Scotland & 0 & 99 & 1,953 & 2,052 & 0.024 & 1 & 84 & 1,919 & 2,004 & 0.021 & $1.14(0.85-1.54)$ & 0.37 \\
\hline Portugal & 0 & 1 & 198 & 199 & 0.003 & 0 & 7 & 179 & 186 & 0.019 & $0.13(0.02-1.06)$ & 0.06 \\
\hline Germany & 0 & 9 & 243 & 252 & 0.018 & 0 & 27 & 1,060 & 1,087 & 0.012 & $1.44(0.68-3.13)$ & 0.34 \\
\hline Combined & 0 & 285 & 6,571 & 6,856 & 0.021 & 3 & 397 & 9,691 & 10,091 & 0.020 & $1.04(0.89-1.22)$ & 0.63 \\
\hline \multicolumn{13}{|l|}{ (B) rs148744804 } \\
\hline England/Wales & 0 & 8 & 3,658 & 3,666 & 0.0011 & 0 & 13 & 6,127 & 6,140 & 0.0011 & $1.03(0.43-2.49)$ & 0.95 \\
\hline Germany & 0 & 0 & 252 & 252 & 0.0000 & 0 & 0 & 1,087 & 1,087 & 0.0000 & NA & NA \\
\hline Combined & 0 & 17 & 6,839 & 6,856 & 0.0012 & 0 & 20 & 10,071 & 10,091 & 0.0010 & $1.14(0.58-2.24)$ & 0.71 \\
\hline
\end{tabular}

$\mathrm{NA}=$ not applicable; $\mathrm{MAF}=$ minor allele frequency; $\mathrm{OR}=$ odds ratio; $\mathrm{Cl}=$ confidence interval. $\mathrm{AA}, \mathrm{AB}$ and $\mathrm{BB}$ denote minor homozygote, heterozygote and major homozygote genotypes respectively. Meta-analysis: $P_{\text {het }}=0.36, I^{2}=9.3 \%$. Meta-analysis: $P_{\text {het }}=0.87, I^{2}=0 \%$.

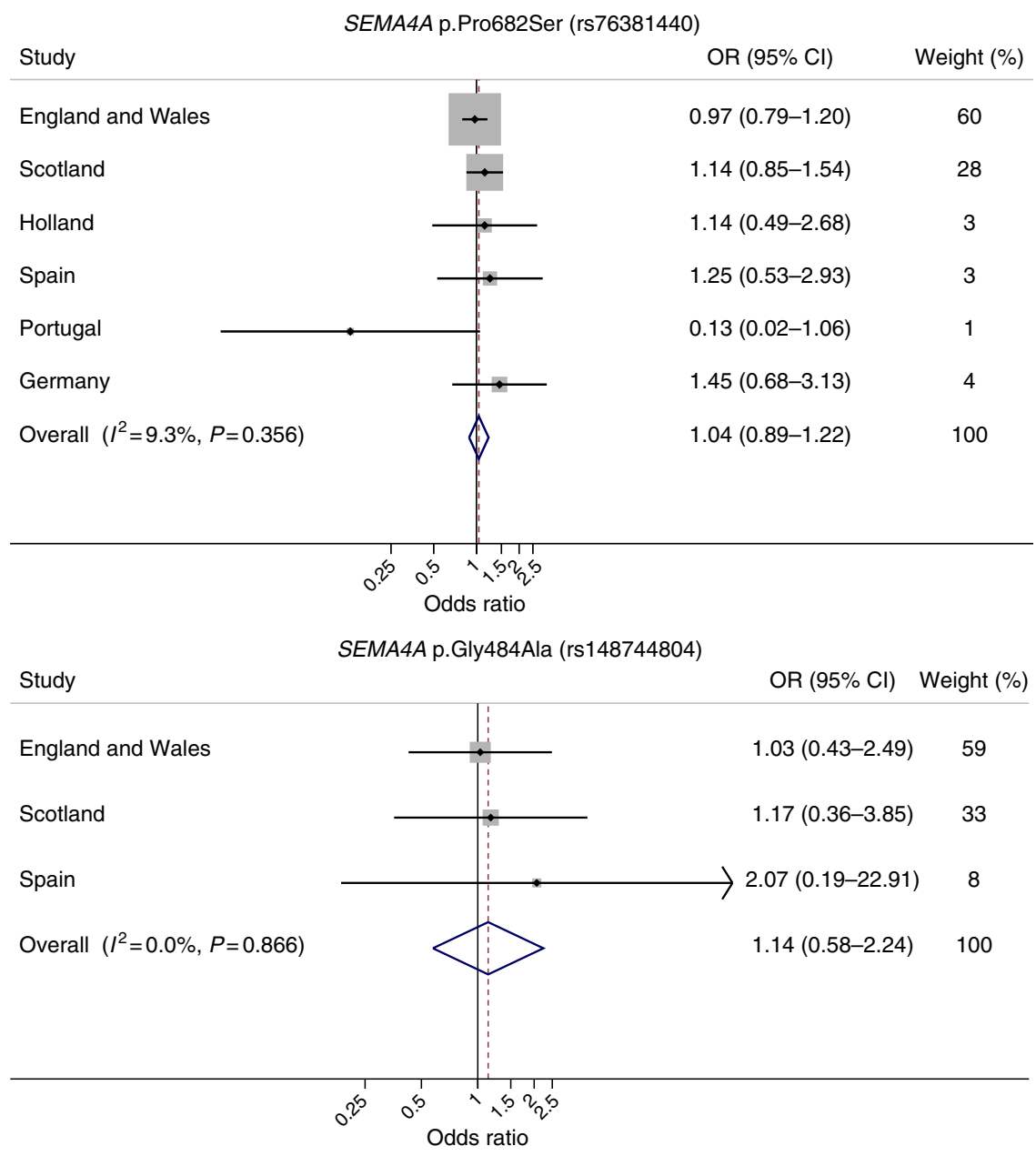

Figure 1 | Forest plot of association between rs148744804 and rs76381440 SEMA4A genotypes and colorectal cancer risk. Studies were weighted according to the inverse of the variance of the log of the OR calculated by unconditional logistic regression. Meta-analysis under a fixed-effects model was conducted using standard methods. Cochran's $Q$ statistic to test for heterogeneity and the $L^{2}$ statistic to quantify the proportion of the total variation due to heterogeneity were calculated. Horizontal lines indicate $95 \%$ confidence intervals (Cls). Boxes indicate odds ratio (OR) point estimate; its area is proportional to the weight of the study. Diamond (and broken line) indicates overall summary estimate, with $\mathrm{Cl}$ given by its width. Unbroken vertical line indicates null value $(\mathrm{OR}=1.0)$. 
interval $[\mathrm{CI}]: 0.58-2.24, P_{\text {heterogenity }}=0.87, I^{2}=0 \%$ and $\mathrm{OR}=$ 1.04, 95\% CI: $0.89-1.22, P_{\text {heterogenity }}=0.36, I^{2}=9 \%$, respectively; Fig. 1). Principal component analysis adjustment had no impact on findings.

Following on from these analyses we examined the mutational spectra of SEMA4A in 1,006 familial early-onset CRC cases $(\geq 1$ first-degree relative with CRC, <56yrs; 158 with FCCTX) from the National Study of Colorectal Cancer Genetics (NSCCG) and 1,609 1958 BC controls sequenced using Illumina Truseq exome capture in conjunction with HiSeq2000 technology (Supplementary Figure 2, Supplementary Table 2). Over 99\% of the SEMA4A transcript was covered at a depth greater than 10 reads (average coverage $38 \times$, Supplementary Figure 2). We identified 28 protein changing variants in 354 samples. Of these variants, there were three unique frameshifts present in two controls and one case. Overall, 13\% of CRC cases (16\% FCCTX) had a protein changing variant in SEMA4A in comparison with $14 \%$ of controls.

There are a number of possible explanations for the disparity between our findings and those reported by Sill and co-workers ${ }^{1}$. Population stratification can lead to spurious associations, and this is especially important with rare variants. The study by Sill and co-workers did not account for this and indeed in comparing the frequency of rs14874408 included cases from both the US and Germany, whereas we ensured ancestral comparability of case patients and control subjects from single nucleotide polymorphism genotypes, thereby excluding this as a source of bias. Generalisability is central to establishing a mutation-phenotype relationship. The evidence for p.Val78Met being causative in FCCTX is based on incomplete segregation in the family reported by Schulz et al. Hence there is the issue of type 1 error.

Much of the missing heritability of CRC is likely to be a result of high/moderate penetrance mutations and rare variants. As illustrated by the recent identification of POLE and POLD1 as a cause of familial $\mathrm{CRC}^{4}$, this class of susceptibility is especially important in understanding cancer biology and for clinical practice. Hence there is a strong rationale for seeking to identify additional such genes. Given the high frequency of deleterious mutations carried by the healthy population, it is becoming increasingly clear that robust and well-powered studies are required to prevent erroneous findings from exome-sequencing projects being asserted to be causal of disease.

In conclusion, in this well-powered study, we find no evidence to support variation in SEMA4A as a determinant of CRC risk. Given that a priori SEMA4A is not a strong candidate CRC predisposition gene, having previously been shown to cause eye disease, we feel that caution should be exercised before SEMA4A is considered as a cause of CRC.

\section{References}

1. Schulz, E. et al. Germline variants in the SEMA4A gene predispose to familial colorectal cancer type X. Nat. Commun. 5, 5191 (2014).

2. Kinnersley, B. et al. Re: Role of the oxidative DNA damage repair gene OGG1 in colorectal tumorigenesis. J. Natl. Cancer Inst. 106, dju086 (2014).

3. Timofeeva, M. N. et al. Recurrent coding sequence variation explains only a small fraction of the genetic architecture of colorectal cancer. Sci. Rep. 5, 16286 (2015).

4. Palles, C. et al. Germline mutations affecting the proofreading domains of POLE and POLD1 predispose to colorectal adenomas and carcinomas. Nat. Genet. 45, 136-144 (2013).

\section{Acknowledgements}

The Leeds collection was supported by Cancer Research UK programme award (C588/ A10589). MGD's laboratory is supported by a Cancer Research UK programme grant (C348/A12076). Funding for quality control of the 1958 Birth Cohort exome chip data was through the Wellcome Trust Case Control Consortium. The Leiden study was supported by the Dutch Cancer Society (UL2010-4656) and the Association for International Cancer Research (10-0619). The Portuguese study was partly funded by the Genetic study of Common Hereditary Bowel Cancers in Hispania and the Americas (CHIBCHA) FP7 project and by Liga Portuguesa Contra o Cancro, Núcleo Regional do Norte. Work in IT's laboratory is supported by funding from the Oxford National Institute for Health Research Biomedical Research Centre and core funding to the Wellcome Trust Centre for Human Genetics from the Wellcome Trust (090532/Z/09/Z). Work in the laboratory of RSH is supported by funding from Cancer Research UK (C1298/A8362, supported by the Bobby Moore Fund). Additional funding is provided by the National Health Service through the Biological Research Centre of the National Institute for Health Research at the Royal Marsden Hospital National Health Service Trust. BK is in receipt of a PhD Studentship from the Institute of Cancer Research and received sponsorship from the Sir John Fisher Foundation. SCB is supported by a contract from the Fondo de Investigación Sanitaria (CP 03-0070). Centro de Investigación Biomédica en Red en el Área temática de Enfermedades Hepáticas y Digestivas (CIBERehd) and Centro de Investigación Biomédica en Red de Enfermedades Raras (CIBERER) are funded by the Instituto de Salud Carlos III. This work was supported by grants from the Fondo de Investigación Sanitaria/FEDER (PI14/00173, PI14/00230) and Asociación Española contra el Cáncer (Fundación Científica GCB13131592CAST). We are sincerely grateful to all patients participating in this study who were recruited in 25 (EPICOLON 1) and 14 (EPICOLON 2) Spanish hospitals as part of the EPICOLON project. We are also indebted to the Spanish National Genotyping Center (Centro Nacional de Genotipado, 1 Instituto de Salud de Salud Carlos III) and the Biobanks of Hospital Clínic-IDIBAPS for technical help. The work was carried out (in part) at the Esther Koplowitz Centre, Barcelona. This publication is supported by COST Action BM1206. We acknowledge use of control genotypes from samples from the Lothian Birth Cohort and Generation Scotland. The 1958 Birth Cohort exome chip data was QCd by Kathy Stirrups. Data sharing was organized by the UK Exome-chip consortium. Exomechip design information: http://genome.sph.umich.edu/wiki/Exome_Chip_Design. Exome variant server: http://evs.gs.washington.edu/EVS/. The 1958 Birth Cohort control exomes include the ICR1000 dataset generated by Professor Nazneen Rahman's Team in the Division of Genetics \& Epidemiology at The Institute of Cancer Research, London. This control set is available to download from EGA: reference EGAD00001001021.

\section{Author contributions}

R.S.H. and B.K. designed the study. R.S.H., D.C. and B.K. drafted the manuscript, with contributions from all authors. For the exome-chip project, B.K., S.B., M.N.T., S.C.-B., S.M.F., A.F., J.H., K.H., R.M.W.H., E.N., C.P., M.P., C.R.P., C.S., M.R.,T., H.W., T.W., D.T.B., I.T., M.G.D. and R.S.H. were involved in study design, sample collection and assembly, data collection and quality control and/or data analysis for their respective centres. For the exome-sequencing project B.K., D.C., S.E.D., M.F. and R.S.H. were involved in study design and/or data analysis.

\section{Additional information}

Supplementary Information accompanies this paper at http://www.nature.com/ naturecommunications

Competing financial interests: The authors declare no competing financial interests.

Reprints and permission information is available online at http://npg.nature.com/ reprintsandpermissions/

How to cite this article: Kinnersley, B. et al. Correspondence: SEMA4A variation and risk of colorectal cancer. Nat. Commun. 7:10611 doi: 10.1038/ncomms10611 (2016).



This work is licensed under a Creative Commons Attribution 4.0 International License. The images or other third party material in this article are included in the article's Creative Commons license, unless indicated otherwise in the credit line; if the material is not included under the Creative Commons license, users will need to obtain permission from the license holder to reproduce the material. To view a copy of this license, visit http://creativecommons.org/licenses/by/4.0/ 